\title{
STUDY OF GLOBAL SOLUTIONS OF PARABOLIC EQUATIONS VIA $A$ PRIORI ESTIMATES III. EQUATIONS OF $p$-LAPLACIAN TYPE
}

\author{
GARY M. LIEBERMAN \\ Department of Mathematics, Iowa State University \\ Ames, Iowa 50011, U.S.A. \\ E-mail: lieb@pollux.math.iastate.edu
}

Introduction. In this work, we continue the series $[9,10]$ which is based on Fila's approach to boundedness of global solutions to nonlinear parabolic equations [3, 4]. Now we examine equations of the form

$$
u_{t}=\operatorname{div} A(D u)+f(u, D u)
$$

for a vector function $A$ with $\partial A(p) / \partial p$ a positive definite matrix. Our model case will be $A(p)=|p|^{m-2} p$, which corresponds to the so-called $p$-Laplacian (although we are using $m$ in place of $p$ ). Under various hypotheses connecting $A$ and $f$, we shall show that global solutions of $(0.1)$ with zero boundary values in a cylinder $\Omega \times(0, \infty)$ with smooth cross-section $\Omega$ are actually bounded in $L^{\infty}$. For this boundedness, we will assume that $f$ depends only on $u$, but our major focus is really on an intermediate result, which we formulate here for a special case.

THEOREM 0.1. Let $u$ be a solution of (0.1) in a $C^{2}$ domain $\Omega \subset \mathbb{R}^{n}$ with

$$
u=0 \quad \text { on } \partial \Omega \times(0, \infty)
$$

for $A(p)=|p|^{m-2} p$, with $2<n<m$, suppose there are positive functions $C_{0}$ and $C_{1}$ such that

$$
|f(z, p)| \leq C_{0}(|z|)+\theta|p|^{(n m+m-n) / n}+C_{1}(\theta)
$$

for all $(z, p) \in \mathbb{R} \times \mathbb{R}^{n}$ and all $\theta>0$, and set

$$
E(t)=\int_{\Omega \times\{t\}}|D u|^{m} d x
$$

1991 Mathematics Subject Classification: Primary 35B45, 35K55, 35K57

The paper is in final form and no version of it will be published elsewhere. 
If there are positive constants $\tau, \sigma, B$ and $k$ such that $\tau>\sigma, B>k$, and

$$
E(\tau)=B, \quad E(\sigma)=k, \quad E(t) \leq B \quad \text { for } t \in[\sigma, \tau]
$$

then

$$
\tau-\sigma \geq \eta
$$

for some positive constant $\eta\left(B, k, C_{0}, C_{1}, m, n, \Omega\right)$.

A similar result will be proved when the inequalities $2<n<m$ fail to hold, but then condition (0.3) must be suitably modified. If $1<m<n$ and $n>2$ instead of $n<m$ and if

$$
|f(z, p)| \leq \theta|z|^{(n m+m-n) /(n-m)}+\theta|p|^{(n m+m-n) / n}+C_{1}(\theta)
$$

replaces (0.3), then (0.5) implies (0.6). (One must also assume $m>2 n /(n+2)$ for the global boundedness result; see the remarks after Lemma 2.2.) For $n=m>2$, (0.3) is replaced by

$$
|f(x, z)| \leq \exp \left(\theta|z|^{n /(n-1)}\right)+\theta|p|^{n}+C_{1}(\theta)
$$

For $n=2$, all these implications hold with $\theta|p|^{(n m+m-n) / n}$ replaced by $|p|^{(3 / 2) m-1-\theta}$. Finally, if $1=n<m$, then (0.5) implies (0.6) if (0.3) is replaced by

$$
|f(x, z)| \leq C_{0}(|z|)+C_{1}|p|^{m}
$$

for some constant $C_{1}$.

Most of our effort will be to prove that (0.5) implies (0.6) for a suitable choice of $E$ based on the hypotheses on $A$ and $f$ (and, in some cases, that $B$ and $k$ are sufficiently far apart). We also examine the related problem with boundary condition

$$
A(D u) \cdot \gamma+\psi(u)=0 \quad \text { on } \partial \Omega \times(0, T),
$$

where $\gamma$ denotes the unit inner normal to $\partial \Omega$.

In addition to structure modelled on $A(p)=|p|^{m-2} p$ or $A(p)=G(|p|) p$ for some function $G$, we also consider a structure typified by defining $A^{i}(p)=\left|p_{i}\right|^{m_{i}-2} p_{i}$ for suitable constants $m_{i}$ or, more generally, $A^{i}(p)=G_{i}\left(\left|p_{i}\right|\right) p_{i}$ for appropriate functions $G_{i}$. Reasoning by analogy with other results concerning such problems, it seems natural to assume also that each $G_{i}$ is a Young's function so that the theory of Orlicz spaces [7] can be used. Although much of our analysis is closely related to that theory, our slightly more general assumptions are a better setting for the problem at hand. Indeed, many of our technical lemmata are obvious when $G$ (or each $G_{i}$ ) is a power function, but there seem to be no previous appearances of them in print.

We begin in Section 1 with a second derivative estimate which is needed to handle the correct growth condition on $f$ with respect to the gradient. Section 2 discusses a suitable generalization of Theorem 0.1 , and its corresponding global boundedness conclusion, in case $A$ grows rapidly at $\infty$, as in Theorem 0.1 with $m>n$. The case of slower growth $(m<n$ in Theorem 0.1) is covered in Section 3. The borderline case (which corresponds to $m=n$ in Theorem 0.1 ) is the topic of Section 4 . Section 5 deals with boundary condition (0.7), and Section 6 discusses some extensions of the results to more general siutations. 
1. A second derivative estimate. When dealing with the equation $u_{t}=\Delta u+$ $f(u, D u)$ in [9], we used the $L^{2}$ Schauder theory to estimate terms in $E^{\prime}(t)$ arising from the gradient dependence of $f$. If the Laplacian is replaced by the $p$-Laplacian, the corresponding inequality is not part of the usual theory of such operators. In this section, we prove such a result, which is similar to [6, Theorem 3.1.1.1] of Grisvard for studying global second derivative estimates of solutions of linear elliptic equations in convex domains.

Theorem 1.1. Let $\Omega$ be a bounded $C^{2}$ domain in $\mathbb{R}^{n}$, and let $A$ be a $C^{2} \mathbb{R}^{n}$-valued function defined on $\Omega \times \mathbb{R}^{n}$. Suppose also that there are positive constants $\delta \leq 1, \Lambda, M$, and $F$ and $a C^{1}$, increasing function $g$ such that

$$
\begin{gathered}
\delta \leq \frac{\tau g^{\prime}(\tau)}{g(\tau)} \leq F, \\
|A(x, p)|+|p|\left|A_{p}(x, p)\right|+\left|A_{x}(x, p)\right| \leq \Lambda g(|p|), \\
a^{i j}(x, p) \xi^{i} \xi^{j} \geq \frac{g(|p|)}{|p|}|\zeta|^{2}
\end{gathered}
$$

for all $(x, p, \xi) \in \Omega \times \mathbb{R}^{n} \times \mathbb{R}^{n}$ with $|p| \geq M$ and all $\tau \geq M$, where $a^{i j}=\partial A^{i} / \partial p_{j}$. If $u \in C^{2}(\bar{\Omega})$ solves

$$
\operatorname{div} A(x, D u)=b(x) \quad \text { in } \Omega, \quad u=0 \quad \text { on } \partial \Omega,
$$

then

$$
\int_{\Omega}\left(1-\frac{M}{|D u|}\right)_{+}^{2} g^{\prime}(|D u|)^{2}\left|D^{2} u\right|^{2} d x \leq C(\Omega, n, \delta, \Lambda, F) \int_{\Omega}\left[g(|D u|)^{2}+b^{2}\right] d x .
$$

Proof. We note that our hypotheses are invariant under any $C^{2}$ change of independent variable. Therefore, we consider first the case that $\partial \Omega$ is just the set $\left\{x^{n}=0\right\}$. Multiplying the differential equation by $\eta$, an arbitrary smooth function, yields

$$
\int_{\left\{x^{n}=0\right\}} A^{n} \eta d x^{\prime}=\int_{\Omega}\left[-A^{i} D_{i} \eta-b \eta\right] d x .
$$

Now we take $\eta=D_{k} \zeta^{k}$ for $\zeta$ a smooth vector-valued function (and we note that the sum on $k$ goes all the way to $n$ ). Integration by parts yields

$$
\int_{\left\{x^{n}=0\right\}}\left[A^{n} D_{k} \zeta^{k}-A^{i} D_{i} \zeta^{n}\right] d x^{\prime}=\int_{\Omega}\left(D_{k}\left(A^{i}\right)+b \delta_{k}^{i}\right) D_{i} \zeta^{k} d x .
$$

A simple approximation argument shows that this inequality holds if $\zeta \in C^{1}$. In particular, if $\zeta^{k}=0$ for $x^{n}=0$ and $k<n$ and if $\zeta$ has compact support, then

$$
\int_{\left\{x^{n}=0\right\}}\left[A^{n} D_{k} \zeta^{k}-A^{i} D_{i} \zeta^{n}\right] d x^{\prime}=-\sum_{i<n} \int_{\left\{x^{n}=0\right\}} A^{i} D_{i} \zeta^{n} d x^{\prime} .
$$

To proceed, we suppose that $h$ is a nonnegative $C^{1}([M, \infty))$ function with $h(M)=0$ and

$$
\delta-1 \leq \frac{\tau h^{\prime}(\tau)}{h(\tau)} \leq h_{1} \quad \text { on }[M, \infty)
$$

for some positive constant $h_{1}$, and take $\zeta=h(|D u|) \eta^{2} D u$ for $\eta$ a $C^{2}$ function with compact support such that $0 \leq \eta \leq 1$. First we examine the boundary integral. Setting 
$H(\tau)=\tau h(\tau)$ and summing on $i$ only up to $n-1$ yields

$$
\begin{aligned}
\int_{\left\{x^{n}=0\right\}} A^{i} D_{i} \zeta^{n} d x^{\prime}= & \int_{\left\{x^{n}=0\right\}} A^{i}\left(x, 0, \ldots, 0, D_{n} u\right) H^{\prime}\left(D_{n} u\right) D_{i n} u \eta^{2} d x^{\prime} \\
& +\int_{\left\{x^{n}=0\right\}} A^{i}\left(x, 0, \ldots, 0, D_{n} u\right) H\left(D_{n} u\right) \eta D_{i} \eta d x^{\prime} .
\end{aligned}
$$

Now we define $h^{i}(x, \tau)=\int_{0}^{\tau} A^{i}(x, 0, \ldots, 0, \sigma) H^{\prime}(\sigma) d \sigma$ and integrate by parts to see that

$$
\begin{aligned}
\int_{\left\{x^{n}=0\right\}} A^{i}\left(x, 0, \ldots, 0, D_{n} u\right) & H^{\prime}\left(D_{n} u\right) D_{i n} u \eta^{2} d x \\
= & -\int_{\left\{x^{n}=0\right\}}\left[2 h^{i}\left(x, D_{n} u\right) \eta D_{i} \eta+\left(\partial h^{i}(x, D u) / \partial x^{i}\right) \eta^{2}\right] d x^{\prime} .
\end{aligned}
$$

Simple calculations show that

$$
\left|A^{i}\left(x, 0, \ldots, 0, D_{n} u\right) H\left(D_{n} u\right)\right|+\left|h^{i}\left(x, D_{n} u\right)\right|+\left|\partial h^{i} / \partial x^{i}\right| \leq C g(|D u|) H(|D u|),
$$

and hence

$$
-\int_{\left\{x^{n}=0\right\}} A^{i} D_{i} \zeta^{n} d x^{\prime} \leq C \int_{\left\{x^{n}=0\right\}} g(|D u|) H(|D u|) \eta d x^{\prime}
$$

with $C$ depending also on $h_{1}$. Integrating by parts and suppressing the argument $|D u|$ in $g, h$ and $H$, we find that

$$
\begin{aligned}
\int_{\left\{x^{n}=0\right\}} g H \eta d x^{\prime} & =\int_{\Omega}\left[(g H)^{\prime} D_{n}(|D u|) \eta(x)+g H D_{n} \eta(x)\right] d x \\
& \leq C \int_{\Omega}\left(\frac{g h}{(1-|D u| / M)}\left|D^{2} u\right| \eta+|D u| g h|D \eta|\right) d x \\
& \leq \varepsilon \int_{\Omega} \frac{g h}{|D u|}\left|D^{2} u\right|^{2} \eta^{2} d x+C(\varepsilon) \int_{\Omega} \frac{g h|D u|}{(1-M /|D u|)^{2}} d x
\end{aligned}
$$

for any $\varepsilon>0$. Hence, choosing $\varepsilon=\frac{\delta}{4}$ gives

$$
-\int_{\left\{x^{n}=0\right\}} A^{i} D_{i} \zeta^{n} d x^{\prime} \leq \frac{\delta}{4} \int_{\Omega} \frac{g h}{|D u|}\left|D^{2} u\right|^{2} \eta^{2} d x+C \int_{\Omega} \frac{g h|D u|}{(1-M /|D u|)^{2}} d x .
$$

To estimate the interior integral in (1.5), we set $C_{k}^{i}=\partial A^{i} / \partial x^{k}+b \delta_{i}^{k}$ and $g^{k m}=\delta^{k m}-$ $|D u|^{-2} D_{k} u D_{m} u$. It follows from the Cauchy-Schwarz inequality that

$$
\begin{aligned}
\int_{\Omega}\left(D_{k}\left(A^{i}\right)+b \delta_{k}^{i}\right) & D_{i} \zeta^{k} d x \\
= & \int_{\Omega} a^{i j} D_{j k} u D_{i k} u h \eta^{2} d x+\int_{\Omega}|D u| a^{i j} D_{i}(|D u|) D_{j}(|D u|) h^{\prime} \eta^{2} d x \\
& +\int_{\Omega} 2 a^{i j} D_{j k} u D_{k} u h \eta D_{i} \eta d x+\int_{\Omega} h C_{k}^{i} g^{k m} D_{i m} u \eta^{2} d x \\
& +\int_{\Omega} C_{k}^{i} D_{k} u D_{i}(|D u|) h^{\prime} \eta^{2} d x+2 \int_{\Omega} C_{k}^{i} D_{k} u h D_{i} \eta \eta d x
\end{aligned}
$$




$$
\begin{aligned}
& \geq \frac{\delta}{2} \int_{\Omega} a^{i j} D_{i k} u D_{j k} u h \eta^{2} d x-C \int_{\Omega} \eta^{2} \frac{|D u| h}{(1-M /|D u|) g} C_{k}^{i} C_{k}^{i} d x \\
& \quad-C \int_{\Omega} g h|D u||D \eta|^{2} d x
\end{aligned}
$$

with the constants depending also on $h_{1}$. Combining these estimates and using (1.1) and (1.2) now yields

$$
\int_{\Omega} h \eta^{2} g^{\prime}\left|D^{2} u\right|^{2} d x \leq C \int_{\Omega}\left(g^{2}+b^{2}\right) \frac{h|D u|}{(1-M /|D u|)^{2} g} d x .
$$

An easy approximation argument shows that this inequality is valid even if $h$ is only Lipschitz. In particular we can take $h(\tau)=(1-M / \tau)_{+}^{2} g(\tau) / \tau$ and note that $h(\tau) \geq$ $C g^{\prime}(\tau)(1-M / \tau)+$ to see that

$$
\int_{\Omega}\left(1-\frac{M}{|D u|}\right)_{+}^{2} g^{\prime}(|D u|)^{2}\left|D^{2} u\right|^{2} \eta^{2} d x \leq C(\delta, F, \Lambda) \int_{\{|D u|>M\}}\left[g(|D u|)^{2}+b^{2} \eta^{2}\right] d x .
$$

A standard partition of unity argument and conversion to the original coordinates yields (1.4).

The technical details of our proof are modelled on Simon's gradient bounds for nonuniformly elliptic equations [13], and a future work will show how to obtain maximum bounds on the gradient near the boundary without using barrier arguments. Other choices of $h$ can be used to derive (1.4) or modifications of that condition. For example, $h(\tau)=(g(\tau) / \tau)\left(\tau /\left(1+\tau^{2}\right)^{1 / 2}\right)^{1-\delta}$ gives an estimate of an integral of the form $\int_{\Omega} \Phi(|D u|)\left|D^{2} u\right|^{2} d x$ with $\Phi$ vanishing only at zero, but this estimate uses information about the behavior of the coefficients for small values of $|D u|$. For our applications, we only use the following gradient estimate, which is a simple consequence of our second derivative bound.

COROLLARY 1.2. Under the hypotheses of Theorem 1.1, we have

$$
\begin{aligned}
& \left(\int_{\Omega} g(|D u|)^{2 \kappa} d x\right)^{1 / \kappa} \\
& \quad \leq C(\delta, F, g(1), \Lambda, \Omega)\left(\int_{\Omega} b^{2} d x+g(M)^{2}+\left(\int_{\Omega} g(|D u|) d x\right)^{2}\right),
\end{aligned}
$$

where $\kappa=n /(n-2)$ if $n>2$ and $\kappa$ is arbitrary for $n \leq 2$.

Proof. We start with the easily verified inequality

$$
\int_{\{|D u|>2 M\}} g^{2 \kappa} d x \leq 4^{\kappa} \int_{\Omega}\left[\left(1-\frac{M}{|D u|}\right)^{2} g\right]^{2 \kappa} d x .
$$

Since

$$
\left|D\left((1-M /|D u|)^{2} g(|D u|)\right)\right| \leq(2+F)(1-M /|D u|) g /|D u|,
$$


we infer from Theorem 1.1 and the Sobolev inequality that

Next we note that

$$
\left(\int_{\{|D u|>2 M\}} g(|D u|)^{2 \kappa} d x\right)^{1 / \kappa} \leq C\left(\int_{\Omega} b^{2} d x+\int_{\Omega} g(|D u|)^{2} d x\right) .
$$

and infer that

$$
\int_{\{|D u| \leq 2 M\}} g(|D u|)^{2 \kappa} d x \leq g(2 M)^{2 \kappa}|\Omega|
$$

$$
\left(\int_{\Omega} g(|D u|)^{2 \kappa} d x\right)^{1 / \kappa} \leq C\left(\int_{\Omega} b^{2} d x+g(M)^{2}+\int_{\Omega} g(|D u|)^{2} d x\right) .
$$

The proof is completed by applying Hölder's inequality.

In particular, for the $p$-Laplace operator, $g(\tau)=\tau^{p-1}$, so Corollary 1.2 in conjunction with Hölder's inequality gives an estimate for the integral of $|D u|^{(2 p-2) \kappa}$.

2. Rapid growth. We begin with an estimate in case $A(p)$ grows rapidly as $p$ goes to infinity. The model function is $A(p)=|p|^{m-2} p$ for some $m>n$. Also here and below, we define

$$
Q=\Omega \times\left(t_{0}, t_{1}\right), \quad S Q=\partial \Omega \times\left(t_{0}, t_{1}\right),
$$

where $t_{0}<t_{1}$ are positive constants and $\Omega$ is a bounded domain in $\mathbb{R}^{n}$ for some $n \geq 1$ with $\partial \Omega \in C^{2}$.

THEOREM 2.1. Let u solve

$$
u_{t}=\operatorname{div} A(D u)+f(u, D u) \quad \text { in } Q, \quad u=0 \quad \text { on } S Q .
$$

Suppose there are scalar functions $S, g$, and $G$ satisfying

$$
\begin{gathered}
A(p)=\frac{\partial S}{\partial p}(p), \\
G(s)=s g(s), \\
g i s C^{1} \text { and increasing on }(0, \infty), \\
S(p) \geq G(|p|),
\end{gathered}
$$

and

$$
\int_{0}^{\infty} \frac{G^{-1}(\sigma)}{\sigma^{(n+1) / n}} d \sigma<\infty
$$

and such that (1.1) and (1.2) hold. Suppose also that there are an increasing, continuous function $C_{0}$ and a positive decreasing function $C_{1}$ such that

$$
|f(z, p)| \leq C_{0}(|z|)+C_{1}(\theta)+\theta g(|p|)^{1+1 / n}|p|^{1 / n}
$$

for all $\theta>0$ if $n \geq 3$,

$$
|f(z, p)| \leq C_{0}(|z|)+C_{1}(\theta)+C_{2} g(|p|)^{1+\theta}|p|^{\theta}
$$

for some $\theta \in(0,1 / 2)$ if $n=2$, and

$$
|f(z, p)| \leq C_{0}(|z|)+C_{2} G(|p|)
$$


if $n=1$. Finally, define $E$ by

$$
E(t)=\int_{\Omega \times\{t\}} S(D u) d x
$$

Then for any $B>0$, there is a positive constant $C\left(B, C_{0}, C_{1}, g_{0}, \Omega, F, \delta, \Lambda\right)$ for which

$$
E(t) \leq B \quad \text { for } t \in\left[t_{0}, t_{1}\right]
$$

implies

$$
E\left(t_{1}\right)-E\left(t_{0}\right) \leq C\left[t_{1}-t_{0}\right]
$$

Proof. Suppose first that $n \geq 3$ and that $u \in C^{2}\left(\Omega \times\left[t_{0}, t_{1}\right]\right)$. By direct calculation and the divergence theorem,

$$
E^{\prime}(t)=\int_{\Omega \times\{t\}} A(D u) \cdot D u_{t} d x=-\int_{\Omega \times\{t\}}(\operatorname{div} A) u_{t} d x .
$$

From the differential equation for $u$ and Cauchy's inequality, we infer that

$$
E^{\prime}(t) \leq-\frac{1}{2} \int_{\Omega \times\{t\}}(\operatorname{div} A)^{2} d x+\frac{1}{2} \int_{\Omega \times\{t\}} f^{2} d x
$$

Next, we use (2.5), (2.6a), and the Sobolev-Orlicz imbedding theorem [2, Theorem 3.2b] to see that

$$
E^{\prime}(t) \leq-\frac{1}{2} \int_{\Omega \times\{t\}}(\operatorname{div} A)^{2} d x+C(\theta)+\theta \int_{\Omega \times\{t\}} g(|D u|)^{2+2 / n}|D u|^{2 / n} d x
$$

for any $\theta>0 ; C$ also depends, of course, on the same quantities as the constant in (2.8b). This last integral is estimated via Hölder's inequality and Corollary 1.2 to obtain

$$
\begin{aligned}
\int_{\Omega \times\{t\}} g(|D u|)^{2+2 / n}|D u|^{2 / n} d x & \leq\left(\int_{\Omega \times\{t\}} g(|D u|)^{2 \kappa} d x\right)^{1 / \kappa}\left(\int_{\Omega \times\{t\}} g(|D u|)|D u| d x\right)^{2 / n} \\
& \leq C\left(\int_{\Omega \times\{t\}}(\operatorname{div} A)^{2} d x+1\right) .
\end{aligned}
$$

Hence

$$
E^{\prime}(t) \leq\left(C \theta-\frac{1}{2}\right) \int_{\Omega \times\{t\}}(\operatorname{div} A)^{2} d x+C(\theta) \leq C
$$

provided $\theta$ is taken sufficiently small. Integrating this inequality yields $(2.8 \mathrm{~b})$ for smooth $u$. The general case for $n \geq 3$ is recovered via the regularization scheme of [8, pp. 342-343].

The cases $n=1$ and $n=2$ can be handled using the ideas in [9, Section 2 and Lemma 3.2]. We leave the details to the reader.

Note that when $n=1$, we don't need to assume (1.1) and (1.2). The proof of [9, Lemma 2.3] shows that we may assume instead that

$$
|f(z, p)| \leq C_{1}(|z|)+C_{2} \Gamma^{1 / 2}(|p|),
$$

where

$$
\Gamma(\tau)=\tau \int_{0}^{\tau} g(s) g^{\prime}(s) s d s
$$


It follows immediately that if there are positive constants $B>k$ and $\tau>\sigma$ satisfying (0.5), then (0.6) holds with $\eta=(B-k) / C$, where $C$ is the constant from $(2.8 \mathrm{~b})$. In particular, Theorem 0.1 follows.

If we assume that $f$ grows more slowly with respect to $p$ than in Theorem 2.1 , we can consider more general hypotheses on $A$.

THEOREM 2.2. Let $u$ solve (2.1), and suppose that there are scalar functions $S, G, g_{i}$, and $G_{i}(i=1, \ldots, n)$ satsifying conditions (2.2), (2.5), and such that

$$
G_{i}(s)=s g_{i}(s),
$$

each $g_{i}$ is $C^{1}$ and increasing on $(0, \infty)$,

$$
\begin{gathered}
G(s)=\left(\prod_{i=1}^{n} G_{i}(s)\right)^{1 / n}, \\
S(p) \geq \sum_{i=1}^{n} G_{i}\left(\left|p_{i}\right|\right) .
\end{gathered}
$$

Suppose also that there are a continuous increasing function $C_{0}$ and a positive constant $\mathrm{C}_{2}$ such that

$$
|f(z, p)| \leq C_{0}(|z|)+C_{2} S(p)^{1 / 2},
$$

and define $E$ by (2.7). Then for any positive $B$, there is a constant $C\left(B, C_{0}, C_{2}, \Omega\right)$ such that (2.8a) implies (2.8b).

Proof. Computing $E^{\prime}$ as in Theorem 2.1 leads to the estimates

$$
\begin{aligned}
E^{\prime}(t) & \leq-\frac{1}{2} \int_{\Omega \times\{t\}}(\operatorname{div} A)^{2} d x+C_{1}^{2} \int_{\Omega \times\{t\}} S(D u) d x+\int_{\Omega \times\{t\}} C_{0}(|u|)^{2} d x \\
& \leq C_{1}^{2} B+C\left(C_{0}, n\right)|\Omega|
\end{aligned}
$$

by the Sobolev-Orlicz imbedding theorem [15, Corollary 1]. The proof is now completed as before.

We now show how Theorem 2.2 leads to a global boundedness result when $f$ is independent of $p$. Portions of this argument are still valid even if $f$ depends on $p$, but a crucial role is played by the Lyapunov functional $J$ defined in Lemma 2.3 below. Rather than discuss this point here, we refer the reader to $[12,16]$ for more details on the construction of general Lyapunov functionals.

In addition to $f$ being independent of $p$, we assume that $f$ is superlinear. In the present context, this assumption takes the following form: There are positive constants $a_{0}, c_{0}, c_{1}$, and $\varepsilon$ such that

$$
\begin{gathered}
p \cdot A(p) \leq a_{0} S(p)+a_{1}, \\
z f(z) \geq\left(a_{0}+\varepsilon\right) F(z)-c_{1}, F(z) \geq z^{2+\varepsilon}-c_{0},
\end{gathered}
$$

where $F(z)=\int_{0}^{z} f(\sigma) d \sigma$. (For the equation $u_{t}=\Delta u+f(u)$, we have $A(p)=p$, and (2.6a) holds with $a_{0}=1 / 2$ and $a_{1}=0$. In this case, $(2.12 \mathrm{~b})$ is equivalent to the single

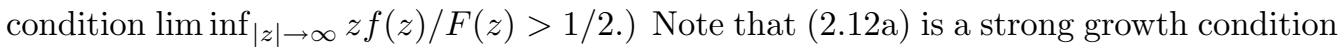


on $S$; if $S(p)=h(|p|)$ for some function $h$, then $h$ satisfies a $\Delta_{2}$-condition as defined in [7, Chapter I, §4]. In particular, $h$ can grow no faster than polynomially.

LEMMA 2.3. Let $n \geq 1$, let $u$ solve

$$
u_{t}=\operatorname{div} A(D u)+f(u) \quad \text { in } \Omega \times(0, \infty), \quad u=0 \quad \text { on } \partial \Omega \times(0, \infty),
$$

and suppose that $f$ and $S$ satisfy (2.12). If $u_{t}, S(D u)$, and $\operatorname{div} A(D u)$ are in $L^{2}(\Omega \times\{T\})$ for any finite $T$, then $\int_{\Omega \times\{t\}} u^{2} d x$ is uniformly bounded for $t \in(0, \infty)$, and

$$
\liminf _{t \rightarrow \infty} E(t)<\infty .
$$

Proof. As in $[3,10]$, we set

$$
M(t)=\frac{1}{2} \int_{\Omega \times\{t\}} u^{2} d x, \quad J(t)=\int_{\Omega \times\{t\}}[S(D u)-F(u)] d x .
$$

After differentiating $M$ with respect to $t$, using the differential equation for $u$ and integrating by parts, we have

$$
M^{\prime}(t)=\int_{\Omega \times\{t\}}[-D u \cdot A(D u)+u f(u)] d x
$$

for almost all $t$. Using (2.12a) and the first inequality of (2.12b), we find that

$$
M^{\prime}(t) \geq \varepsilon \int_{\Omega \times\{t\}} F(u) d x-a_{0} J(t)-\left(a_{1}+c_{1}\right)|\Omega| .
$$

A simple calculation shows that $J^{\prime}(t) \leq 0$, so $J$ is nonincreasing. Taking into account the second inequality of $(2.12 \mathrm{~b})$ and using Hölder's inequality yields $M^{\prime}(t) \geq C M^{1+\varepsilon / 2}-K$ for suitable positive constants $C$ and $K$. As in [4, Lemma 1.2], it follows that $M(t) \leq$ $(K / C)^{2 /(2+\varepsilon)}$ because $u$ exists for all $t$.

Similar calculations (cf. [10, Theorem 2.2]) show that $M^{\prime}(t) \geq \varepsilon E(t)-C$, so $E(t) \rightarrow \infty$ as $t \rightarrow T^{-}$implies that $T$ must be finite, proving (2.14). (Note that these arguments also work in the slow growth case because (2.12a) is the only restriction on $S$.)

Note that $(2.12 \mathrm{~b})$ with $A(p)=|p|^{m-2} p$ implies that $z f(z) \geq C_{2} z^{1+\varepsilon}-C_{3}$ so $(0.3)^{\prime}$ can only be satisfied if $(n m+m-n) /(n-m)>1$, which is equvialent to $m>2 n /(n+2)$.

Combining the results of Theorem 2.2 and Lemma 2.3 with the techniques used in proving [9, Theorem 1.5] gives global boundedness. We outline the procedure because there are some important differences between the situation in $[4,9]$ and the present one.

TheOrem 2.4. Let $n \geq 1$, suppose conditions (2.2), (2.5), (2.9), (2.10) and (2.12) are satisfied, and suppose that $u$ solves (2.13). Suppose also that there is an increasing continuous function $C_{0}$ such that

$$
|f(z)| \leq C_{0}(|z|) .
$$

Then $E(t)$ is uniformly bounded, and $u$ is uniformly bounded in $L^{\infty}$.

Proof. From Lemma 2.3, $E(t)$ cannot tend to infinity as $t \rightarrow \infty$. If $\lim _{t \rightarrow \infty} E(t)$ is finite, we are done. Hence we may assume that there are (finite) constants $k$ and $B$ so 
that

$$
\liminf _{t \rightarrow \infty} E(t)<k<B<\limsup _{t \rightarrow \infty} E(t) .
$$

For any $B$ and $k$ satisfying this inequality, we can find sequences $\left(\sigma_{m}\right)$ and $\left(\tau_{m}\right)$ such that $\sigma_{m}<\tau_{m}<\sigma_{m+1}, \sigma_{m} \rightarrow \infty, E\left(\sigma_{m}\right)=k, E\left(\tau_{m}\right)=B$, and $E(t) \leq B$ for $t \in\left(\sigma_{m}, \tau_{m}\right)$. Since $E\left(\tau_{m}\right)$ is uniformly bounded, it follows from [2, Theorem 3.6] that the sequence $\left(u\left(\cdot, \tau_{m}\right)\right)$ is uniformly bounded and equicontinuous. By taking a suitable subsequence of $\left(\tau_{m}\right)$ (still denoted by $\left(\tau_{m}\right)$ ), we may therefore assume that this sequence converges uniformly to a limit function $v$. Our goal is to show three things about $v$ : first, $v$ is a solution of the limit problem

$$
\operatorname{div} A(D v)+f(v)=0 \quad \text { in } \Omega, \quad v=0 \quad \text { on } \partial \Omega,
$$

second, $\int_{\Omega} S(D v) d x$ is bounded from above by a constant independent of $B$, and, third, this integral is bounded from below by a constant multiple of $B$.

To show that $v$ solves $(2.16)$, we set $u_{m}(X)=u\left(x, t-t_{m}\right)$ and $Q=\Omega \times(0, \eta)$. Then $\int_{Q} S\left(D u_{m}\right) d X \leq B$, so $\int\left|A\left(D u_{m}\right)\right| d X$ is uniformly bounded and hence (by extracting a subsequence as needed) there is a function $v \in L^{1}(Q)$ with $|A(D v)| \in L^{1}(Q)$ and $A\left(D u_{m}\right) \rightarrow A(D v)$ weakly in $L^{1}(Q)$. Therefore

$$
\begin{aligned}
\int_{Q}[A(D v) \cdot D \varphi-f(v) \varphi] d X & =\lim _{m \rightarrow \infty} \int_{Q}\left[A\left(D u_{m}\right) \cdot D \varphi-f\left(u_{m}\right) \varphi\right] d X \\
& =-\lim _{m \rightarrow \infty} \int_{Q} u_{m, t} \varphi d X
\end{aligned}
$$

for any $\varphi \in C_{0}^{1}(\Omega)$.

Now we mulitiply the differential equation for $u$ by $u_{t}$ and integrate by parts to see that

$$
\int_{\Omega \times\left(0, \tau_{m}\right)}\left(u_{t}\right)^{2} d X=J(0)-J\left(\tau_{m}\right) .
$$

Since $J\left(\tau_{m}\right) \geq C(B)$ for some negative constant $C(B)$, it follows that $u_{t} \in L^{2}(\Omega \times(0, \infty))$. Now let $C$ be the constant from Theorem 2.2 and set $\eta=(B-k) / C, t_{m}=\tau_{m}-\eta$ and $Q(m)=\Omega \times\left(t_{m}, \tau_{m}\right)$, noting that $t_{m} \geq \sigma_{m}$. It follows that

$$
\lim _{m \rightarrow \infty} \int_{Q(m)}\left(u_{t}\right)^{2} d X=0,
$$

and hence

$$
\int_{\Omega}\left|u\left(x, \tau_{m}\right)-u\left(x, \tau_{m}-\theta\right)\right| d x \rightarrow 0
$$

uniformly for $\theta \in[0, \eta]$ as $m \rightarrow \infty$. Combined with the equiboundedness of the collection of functions $\left\{u_{m}\right\}$, we find that $v$ is independent of $t$ and hence $v$ is a weak solution of $(2.16)$.

Now we take $v$ as test function in the weak form of (2.16) and use the first inequality 
of $(2.12 \mathrm{~b})$ to see that

$$
\int_{\Omega} A(D v) \cdot D v d x \geq\left(a_{0}+\varepsilon\right) \int_{\Omega} F(v) d x-c_{1}|\Omega|,
$$

and therefore $(2.12 \mathrm{a})$ implies that

or

$$
\int_{\Omega} S(D v) d x \geq\left(1+\frac{\varepsilon}{a_{0}}\right) \int_{\Omega} F(v) d x-\frac{a_{1}+c_{1}}{a_{0}}|\Omega|,
$$

$$
\int_{\Omega} F(v) d x \leq \delta \int_{\Omega} S(D v) d x+k_{1}
$$

for positive constants $\delta$ and $k_{1}$ with $\delta<1$. On the other hand, Fatou's Lemma shows that

$$
\int_{\Omega} S(D v) d x \leq \liminf _{m \rightarrow \infty} \int_{\Omega} S\left(D u\left(x, \tau_{m}\right)\right) d x .
$$

Since the sequence $\left(u\left(\cdot, \tau_{m}\right)\right)$ converges pointwise to $v$ and is uniformly bounded, it follows that $F\left(u\left(\cdot, \tau_{m}\right)\right) \rightarrow F(v)$ in $L^{1}(\Omega)$. Moreover, $J$ is decreasing so

$$
\int_{\Omega} S(D v) d x \leq \lim _{m \rightarrow \infty} J\left(\tau_{m}\right)+\int_{\Omega} F(v) d x \leq J(0)+\int_{\Omega} F(v) d x .
$$

Combining (2.17a) with $(2.17 \mathrm{~b})$ yields, after some rearrangement,

$$
\int_{\Omega} S(D v) d x \leq k_{2}
$$

for $k_{2}=\left(J(0)+k_{1}\right) /(1-\delta)$, which is independent of $B$. Hence

$$
\lim \int_{\Omega} F\left(u\left(\cdot, \tau_{m}\right)\right) d x=\int_{\Omega} F(v) d x \leq C
$$

for some constant independent of $B$. Since also

$$
\lim \int_{\Omega} S\left(D u\left(\cdot, \tau_{m}\right)\right) d x=\lim J\left(\tau_{m}\right)+\lim \int_{\Omega} F\left(u\left(\cdot, \tau_{m}\right)\right) d x \leq J(0)+C,
$$

we conclude that $B \leq J(0)+C$, which means that $E(t)$ is uniformly bounded. The $L^{\infty}$ bound follows from this one by [15, Corollary 1].

The slow growth version of Theorem 2.3 is only slightly more delicate; in place of the uniform $L^{\infty}$ estimates for $u$, we need to observe that the appropriate integrands are bounded by suitable $L^{1}$ functions.

3. Slow growth. In this section, we study problem (2.1) when $A$ grows more slowly than in Section 2. A crucial element of our program is a Sobolev-type inequality involving Orlicz functions. The guiding principle is a straightforward modification of Donaldson and Trudinger's imbedding theorem [2, Theorem 3.2(a)] for Sobolev-Orlicz spaces, but there are some important technical differences. The most important is that we are not interested in results relating Orlicz norms of functions and their derivatives over a suitable domain in $\mathbb{R}^{n}$ but rather the integral of a Young's function composed with the function defined on that domain. In fact, our results do not use the convexity of the Young's function so 
we state them in terms of a slightly different structure, namely (2.3). Unlike the situation in Section 2, we always consider the structure from Theorem 2.1 which uses the single function $g$.

Lemma 3.1. Let $g$ and $G$ satisfy (2.3). Suppose $H$ is a positive $C^{1}(0, \infty)$ function satisfying the differential equation

$$
\left(G^{-1} \circ H\right)(s) \frac{d H}{d s}=H(s)^{(n+1) / n}
$$

for $s>0$. Then

$$
\frac{n+1}{n} \geq \frac{H H^{\prime \prime}}{\left(H^{\prime}\right)^{2}} \geq \frac{1}{n}
$$

Moreover

$$
\frac{d}{d s}\left(\left(H^{\prime}(s)\right)^{2} H(s)^{-1 / n}\right) \leq 2 H^{\prime \prime}(s) g \circ G^{-1}(H(s)) .
$$

Proof. Differentiation of (3.1) followed by a use of (3.1) itself yields

$$
\left(G^{-1}\right)^{\prime}(H)\left(H^{\prime}\right)^{2}+G^{-1}(H) H^{\prime \prime}=\frac{n+1}{n} H^{1 / n} H^{\prime}=\frac{n+1}{n} \frac{\left(H^{\prime}\right)^{2}}{H} G^{-1}(H) .
$$

Since $G$ is increasing, so is its inverse and hence

$$
G^{-1}(H) H^{\prime \prime} \leq \frac{n+1}{n} \frac{\left(H^{\prime}\right)^{2}}{H} G^{-1}(H),
$$

which implies the first inequality of (3.2). For the second, note that $G^{\prime} \geq g$, so

$$
\left(G^{-1}\right)^{\prime}(H)=1 / G^{\prime}\left(G^{-1}(H)\right) \leq 1 / g \circ G^{-1}(H)=G^{-1}(H) / H,
$$

and hence

$$
H^{\prime \prime} \geq\left(\frac{n+1}{n}-1\right) \frac{\left(H^{\prime}\right)^{2}}{H} .
$$

To prove (3.3), we suppress the argument $s$ from $H$ and its derivatives and note that

$$
\frac{d}{d s}\left(\left(H^{\prime}\right)^{2} H^{-1 / n}\right)=H^{\prime} H^{-1 / n}\left[2 H^{\prime \prime}-\frac{1}{n} \frac{\left(H^{\prime}\right)^{2}}{H}\right] \leq 2 H^{\prime \prime} H^{\prime} H^{-1 / n} .
$$

Since (3.1) implies that $g \circ G^{-1}(H)=H^{\prime} H^{-1 / n}$, we infer (3.3).

Note that, even though $G$ and $H$ need not be Young's functions, much of the machinery of Orlicz spaces can still be used. In particular, we define

$$
\|u\|_{H}=\inf \left\{k>0: \int_{\Omega} H\left(\frac{|u|}{k}\right) d x \leq 1\right\},
$$

which is the Luxemburg norm because $H$ is convex. Note that

$$
\int_{\Omega} H\left(\frac{|u|}{\|u\|_{H}}\right) d x=1
$$

if $u$ is bounded. We are now ready to prove our Sobolev inequality. 
LEMma 3.2. Suppose the hypotheses of Lemma 3.1 are satisfied. Let $u \in C^{0,1}(\bar{\Omega})$ with $u=0$ on $\partial \Omega$, and set $\varepsilon_{1}=\min \left\{1 / 8,1 /\|u\|_{H}\right\}$. Then for any $\varepsilon \in\left(0, \varepsilon_{1}\right)$, we have

$$
\int_{\Omega} H^{\prime}(\varepsilon|u|)^{2} d x \leq 4 \varepsilon \int_{\Omega} H^{\prime \prime}(\varepsilon|u|) G(|D u|) d x .
$$

Proof. Without loss of generality, we may assume that $u$ is nonnegative. Then we integrate (3.3) and apply the inequalities of Sobolev and Hölder. Since the constant in Sobolev's inequality is less than 1 (see $[5,(7.28)]$ ), we see that

$$
\int_{\Omega}\left(H^{\prime}(\varepsilon u)\right)^{2} d x \leq C_{1}(\varepsilon) \int_{\Omega} H^{\prime \prime}(\varepsilon u) g \circ G^{-1}(H(\varepsilon u))|D u| d x,
$$

for $C_{1}(\varepsilon)=2 \varepsilon\left[\int_{\Omega} H(\varepsilon u) d x\right]^{1 / n}$. Because $\varepsilon_{1} \leq 1 /\|u\|_{H}$, it follows that $C_{1} \leq 2 \varepsilon$. Then Young's inequality in the form [8, Lemma 1.1(e)] implies that

$$
\int_{\Omega}\left(H^{\prime}\right)^{2} d x \leq 2 \varepsilon\left(\int_{\Omega} H^{\prime \prime} G(|D u|) d x+\int_{\Omega} H^{\prime \prime} H d x\right),
$$

where we suppress the argument $\varepsilon u$ in $H$ and all its derivatives. From (3.2), it follows that

$$
\int_{\Omega}\left(H^{\prime}\right)^{2} d x \leq 2 \varepsilon \int_{\Omega} H^{\prime \prime} G(|D u|) d x+4 \varepsilon \int_{\Omega}\left(H^{\prime}\right)^{2} d x .
$$

The desired result follows from this inequality because $\varepsilon_{1} \leq 1 / 8$.

Now we examine the situation that $A$ has "slow growth" and $f$ is independent of $D u$. Specifically, we assume of $A$ that there are nonnegative constants $g_{0}$ and $g_{1}$, and an increasing function $g$ such that

$$
\begin{array}{ll}
g(s)=g_{1} s^{(n-2) /(n+2)} & \text { for } s \in(0,1), \\
p \cdot A(p) \geq G(|p|)-g_{0} & \text { for all } p \in \mathbb{R}^{n}
\end{array}
$$

(here $G$ is related to $g$ via (2.3a)), and that

$$
\int_{0}^{\infty} \frac{G^{-1}(\tau)}{\tau^{(n+1) / n}} d \tau=\infty
$$

We pause here to make some observations about these conditions. The explicit form of $g$ in (3.5a) is just a convenient technical device to facilitate the proof of Lemma 3.3 below. Moreover, for a given $C^{1}$ function $g^{*}$ defined on $[0, \infty)$, we can find a $C^{1}$ function $g$ satisfying (3.5a) such that $g(s)=g^{*}(s)$ for $s>2$. If (3.5b) holds with $g$ replaced by $g^{*}$ and some constant $g_{0}$, we can always adjust the constants $g_{0}$ and $g_{1}$ to guarantee (3.5b) with $g$. Condition (3.5b) says that $G$ only has to control $p \cdot A$ for large $p$. When $S$ is convex, (3.5b) follows from (2.2) and (2.4). If $G(s)=s^{m}$ for some constant $m$ and all $s>1$, then (3.6) holds if and only if $1 \leq m \leq n$.

The key step in our study of global boundedness is an estimate for $E^{\prime}(t)$, once a suitable choice has been made for $E$. If we take $H$ to be the even extension of the Sobolev conjugate of $G$, so

$$
H^{-1}(s)=\int_{0}^{s} \frac{G^{-1}(\tau)}{\tau^{(n+1) / n}} d \tau
$$


for $s \geq 0$, then $E$ is given by

$$
E(t)=\int_{\Omega \times\{t\}} H(\varepsilon u) d x
$$

with $\varepsilon$ sufficiently small.

LEMma 3.3. Let $u$ be a solution of (2.1) and suppose $E$ is given by (3.7) with $\varepsilon \leq$ $\min \left\{1 / 8,1 /\|u\|_{H}\right\}$. Suppose also that there is an decreasing function $C_{1}$ such that

$$
f(z, p) \operatorname{sgn} z \leq C_{1}(\theta)+\theta\left|H^{\prime}(\theta z)\right|
$$

for all real $z$ and all $\theta \in(0,1)$. If (3.5) and (3.6) hold, then there is a constant $C$ determined only by $C_{1}(\varepsilon),|\Omega|, g_{0}, g_{1}$, and $h=G^{-1}\left(g_{1}^{(n+2) / n} / 4\right)$ such that (2.8a) implies $(2.8 \mathrm{~b})$.

Proof. As before, we may assume $u$ to be $C^{2}$, and then we only need to estimate $E^{\prime}$. By direct calculation,

$$
\begin{aligned}
E^{\prime}(t) & =\varepsilon \int_{\Omega \times\{t\}} H^{\prime}(\varepsilon u) u_{t} d x=\varepsilon \int_{\Omega \times\{t\}}\left[H^{\prime} \operatorname{div} A(D u)+H^{\prime} f\right] d x \\
& =-\varepsilon^{2} \int_{\Omega \times\{t\}} H^{\prime \prime} D u \cdot A d x+\varepsilon \int_{\Omega \times\{t\}} H^{\prime} f d x .
\end{aligned}
$$

Now we use (3.8) with $\theta=\varepsilon$ and then Lemma 3.2 to infer that

$$
\begin{aligned}
E^{\prime}(t) & \leq \varepsilon^{2}\left(-\int_{\Omega \times\{t\}} H^{\prime \prime} D u \cdot A d x+\int_{\Omega \times\{t\}}\left(H^{\prime}\right)^{2} d x+C_{1}(\varepsilon)|\Omega|\right) \\
& \leq \varepsilon^{2}\left(-\frac{1}{2} \int_{\Omega \times\{t\}} H^{\prime \prime} G(|D u|) d x+g_{0} \int_{\Omega \times\{t\}} H^{\prime \prime} d x\right)+C .
\end{aligned}
$$

Since $H(\tau) \rightarrow \infty$ as $\tau \rightarrow \infty$, we can use (3.2) to see that

$$
g_{0} \int_{\Omega \times\{t\}} H^{\prime \prime} d x \leq 2 \int_{\left\{H>g_{0}\right\}}\left(H^{\prime}\right)^{2} d x+\int_{\left\{H \leq g_{0}\right\}} H^{\prime \prime} d x .
$$

To estimate the second integral on the right hand side of this inequality, we note that $H(1)=g_{1}^{(n+2) / n} / 4=G(h)$. Therefore, on $\left\{1<\varepsilon|u|, H \leq g_{0}\right\}$, we have

$$
H^{\prime \prime} \leq 2\left(H^{\prime}\right)^{2} / H=\frac{2 H^{1+2 / n}}{h^{2}} \leq \frac{2 H^{1+2 / n}\left(H^{-1}\left(g_{0}\right)\right)}{h^{2}}=\frac{2 g_{0}^{(n+2) / n}}{h^{2}} .
$$

It follows that

$$
g_{0} \int_{\Omega \times\{t\}} H^{\prime \prime} d x \leq 2 \int_{\Omega \times\{t\}}\left(H^{\prime}\right)^{2} d x+\left[g_{0} g_{1}^{(n+2) / n}+2 g_{0}^{(n+2) / n} h^{-2}\right]|\Omega| .
$$

Using this inequality in (3.9) and applying Lemma 3.2 yields (2.8b).

Note that $E$ plays two different roles in Section 2, which now must be played by two functions denoted by $E$ and $U$. We use $U$ to measure how large the solution $u$ is and how fast it changes. In seeing how fast $U$ (and hence $u$ ) changes, we introduce a comparable function $E$ whose derivative is easily estimated. As we shall see in the proof 
of Theorem 3.4, the correct quantity for $U$ is $\|u\|_{H}$, while $E$ is defined by (3.7). Note also that the variational hypothesis (2.2) is not used in this case.

THeOrem 3.4. Let $A$ and $g$ satisfy (2.3), (3.5) and (3.6), suppose that $f$ satisfies (3.8), suppose $u$ solves $(2.1)$, and set $U(t)=\|u(\cdot, t)\|_{H}$. If $B$ and $k$ are positive constants with $B>\max \{k, 8\}$, and if $\tau>\sigma$ are positive constants with

$$
U(\tau)=B, \quad U(\sigma)=k, \quad U(t) \leq B \quad \text { for } t \in(\sigma, \tau),
$$

then there is a positive constant $\eta$ determined only by $B, k, \Omega, C_{1}(1 / B), g_{0}, g_{1}, n$, and $h$ such that (0.6) is satisfied.

Proof. Taking $\varepsilon=1 / B$, we see that

$$
1-\int_{\Omega \times\{\sigma\}} H(\varepsilon u) d x=\int_{\Omega \times\{\tau\}} H(\varepsilon u) d x-\int_{\Omega \times\{\sigma\}} H(\varepsilon u) d x \leq C[\tau-\sigma] .
$$

Because $H^{\prime \prime} \geq 0$, we have $H(\varepsilon u) \leq(\varepsilon k) H(u / k)$ and therefore $1-(k / B)=1-\varepsilon k \leq$ $C[\tau-\sigma]$, which implies (0.6).

When $f$ grows more rapidly with respect to $p$, we must modify Lemma 3.3 to obtain a hybrid of the conditions in Theorem 3.4 and those in Section 2.

LEMma 3.5. Suppose there are scalar functions $S, g$, and $G$ and a positive constant $g_{0}$ such that (2.2), (2.3), and (3.5) are satisfied. Let $u$ be a solution of (2.1) and define E by

$$
E(t)=\int_{\Omega \times\{t\}} S(D u) d x+\lambda \int_{\Omega \times\{t\}} H(\varepsilon u) d x
$$

for some positive constants $\varepsilon$ and $\lambda$. Suppose also that $\Omega \subset \mathbb{R}^{n}$ with $n \geq 3$ and that there is a positive decreasing function $C_{1}$ such that

$$
|f(z, p)| \leq C_{1}(\theta)+\theta\left|H^{\prime}(\theta z)\right|+\theta g(|p|)^{1+1 / n}|p|^{1 / n}
$$

for all real $z$ and all $\theta \in(0,1)$. If there is a constant $B$ such that

$$
\int_{\Omega \times\{t\}} G(|D u|) d x \leq B,
$$

and if $\varepsilon \leq 1 /\left(8 B^{1 / n}\right)$, then there is a constant $C\left(B, C_{1}, \varepsilon, \lambda,|\Omega|, g_{0}, g_{1}, n, h\right)$ such that (2.8b) holds.

Proof. Let us define $\Gamma$ and $\Phi$ by $\Gamma(\tau)=G(\tau) / B$ and

$$
\Phi^{-1}(\tau)=\int_{0}^{\tau} \frac{\Gamma^{-1}(s)}{s^{1+1 / n}} d s .
$$

From the Sobolev-Orlicz imbedding theorem [2, Theorem 3.4(b)], we have $\|u\|_{\Phi} \leq 1$ because the constant $\gamma$ in that inequality is $n^{1 / 2}$. It is a simple matter to check that $\Phi(\tau)=H\left(\tau / B^{1 / n}\right) / B$, so the proof of Lemma 3.2 gives

$$
\int_{\Omega \times\{t\}}\left(H^{\prime}\right)^{2} d x \leq 4 \varepsilon_{1} \int_{\Omega \times\{t\}} H^{\prime \prime} G(|D u|) d x
$$


for any $\varepsilon_{1} \leq 1 / 8$ provided $\varepsilon=\varepsilon_{1} / B^{1 / n}$. Hence the terms obtained from differentiating the first integral in $E$ with respect to $t$ are estimated as in Section 2 while those from the second integral are estimated as in Lemma 3.3.

With $E$ defined by (3.11), the analog of Theorem 3.4 is easier because we don't need to take $B>8$.

Theorem 3.6. Let $A$ and $g$ satisfy (1.1), (1.2), (2.2), (2.3), (2,4), (3.5) and (3.6), suppose that $f$ satisfies (3.12), suppose u satisfies (2.1), and set

$$
U(t)=\int_{\Omega \times\{t\}} S(D u(x, t)) d x
$$

If $B$ and $k$ are positive constants with $B>k$, and if $\tau>\sigma$ are positive constants satisfying (3.10), then there is a positive constant $\delta$ determined only by $B, k, \Omega, C_{1}(1 / B), g_{0}, g_{1}$, $n$, and $h$ such that (0.6) holds.

Proof. Now we use Lemma 3.5 with $0<\varepsilon<\varepsilon_{0}$ and $\lambda=\min \{1,(B-k) / 2\}$. We then have

$$
B-k=U(\tau)-U(\sigma) \leq E(\tau)-E(\sigma)+\lambda \int_{\Omega \times\{\tau\}} H(\varepsilon u) d x .
$$

From our choice of $\varepsilon$ and $\lambda$, it follows that $B-k \leq E(\tau)-E(\sigma)+\frac{1}{2}(B-k) \leq$ $C[\tau-\sigma]+\frac{1}{2}(B-k)$. Rearranging this inequality gives $(0.6)$ with $\eta=1 /(2 C)$.

As previously indicated, the case $n=2$ is handled by using the techniques of $[9$, Lemma 3.2] provided the term $\theta g(|p|)^{1+1 / n}|p|^{1 / n}$ is replaced by $g(|p|)^{1-\theta+1 / n}|p|^{(1 / n)-\theta}$. Because of (1.1), we have $G^{-1}(\sigma) \leq C \sigma^{1-\delta}$ for $\sigma \geq 1$, so (2.2) is always satisfied when $n=1$.

4. The borderline case. The results of Section 3 rely on the specific form of the Sobolev-Orlicz embedding theorem. The Sobolev conjugate function $H$ of $G$ is used to bound the function $f$ in our partial differential equation, and the precise form of this conjugate is used, via Lemmata 3.1 and 3.2 , to control terms in the expression for $E^{\prime}$. It is well-known that a better embedding theorem is valid in case $G(s)=s^{n}$, where $n \geq 2$ and $\Omega \subset \mathbb{R}^{n}$. (The behavior of $G$ for small $s$ is not relevant here.) In this section, we show how to take advantage of the better embedding.

THEOREM 4.1. Let $n \geq 3$, and let $u$ solve (2.1). Suppose there is a scalar function $S$ satisfying (2.2) and (2.4) with $G(s)=s^{n}$. Suppose also that there is a positive decreasing function $C_{1}$ such that

$$
|f(z, p)| \leq C_{1}(\theta) \exp \left(\theta|z|^{n /(n-1)}\right)+\theta|p|^{n}
$$

for all $\theta>0$, and define $E$ by (2.7). Then for any $B>0$, there is a positive constant $C\left(B, C_{0}, C_{1}, g_{0}, \Omega, F, \delta, \Lambda\right)$ for which (2.8a) implies (2.8b).

Proof. We follow the proof of Theorem 2.1. The only difference is in the estimate of $\int f^{2} d x$. We have

$$
\int_{\Omega \times\{t\}} f^{2} d x \leq C(\theta)+\theta \int_{\Omega \times\{t\}}|D u|^{2 n} d x+C_{1}(\theta) \int_{\Omega \times\{t\}} \exp \left(2 \theta|u|^{n /(n-1)}\right) d x,
$$


and the first integral on the right is estimated as before. From the embedding theorem [14, Theorem 2], we know there is a constant $C(n)$ such that if $k$ is a positive constant with

then

$$
k \geq C(n)\left(\int_{\Omega \times\{t\}}|D u|^{n} d x\right)^{1 / n},
$$

$$
\int_{\Omega \times\{t\}} \exp \left(|u / k|^{n /(n-1)}\right) d x \leq 1 .
$$

Using this implication with $k=1 /(2 \theta)$ and $\theta \leq B^{1 / n} /(2 C(n))$ gives $\int_{\Omega \times\{t\}} t f^{2} d x \leq C$ provided $\theta$ is sufficiently small, and then the proof is completed as in Theorem 2.1.

For $n=2$, we must modify (4.1) by using $|p|^{2-\theta}$ in place of $\theta|p|^{n}$. Full details may be found in [9]. Note that the proof of Theorem 4.1 is valid as long as there is a function $H$ satisfying the algebraic inequality

$$
|f(z, p)| \leq C_{1}(\theta) H(\theta|z|)^{1 / 2}+\theta g(|p|)^{1+1 / n}|p|^{1 / n}
$$

and the Sobolev inequality $\|u\|_{H} \leq C\|D u\|_{G}$. The author is currently unaware of situations in which these inequalities are satisfied which do not fall into the cases discussed in Theorem 4.1 or the previous sections of this paper.

5. Nonlinear boundary conditions. In this section we show how to modify our arguments to cover the problem

$$
u_{t}=\operatorname{div} A(D u) \quad \text { in } Q, \quad A(D u) \cdot \gamma+f(u)=0 \quad \text { on } S Q,
$$

with $Q$ and $S Q$ as before, where $\gamma$ denotes the unit inner normal to $\partial \Omega$.

The fast growth case is handled very much like its analog in [9].

LEMMA 5.1. Let $u$ be a solution of (5.1). Suppose conditions (2.2)-(2.5) are satisfied. Suppose also that there is a $C^{1}$ function $C_{1}$ such that $G \circ g^{-1}(|f(z)|) \leq C_{1}(|z|)$ and set

$$
E(t)=\int_{\Omega \times\{t\}}\left[S(D u)-f(u) D u \cdot \gamma+2 C_{1}(|u|)\right] d x .
$$

Then for any positive constant $B$, there is a constant $C=C\left(C_{1}, B, \Omega, g_{0}\right)$ such that

$$
\int_{\Omega \times\{t\}}[G(|D u|)+G(u)] d x \leq B
$$

implies (2.8b).

Proof. Suppose first that $f$ is $C^{1}$ and that $u$ is $C^{2}$. Then

$$
\begin{aligned}
E^{\prime}(t) & =\int_{\Omega \times\{D\}}\left[u_{t} \cdot A(D u)-f(u) D_{t} u \cdot \gamma-u_{t} f^{\prime}(u) D u \cdot \gamma+2 C_{1}^{\prime}(u) u_{t}\right] d x \\
& =\int_{\Omega \times\{t\}}\left[D u_{t} \cdot(A(D u)-f \gamma)-u_{t} f^{\prime}(u) D u \cdot \gamma+2 C_{1}^{\prime}(u) u_{t}\right] d x \\
& =\int_{\Omega \times\{t\}}-u_{t}\left[\operatorname{div}(A(D u)-f(u) \gamma)+f^{\prime}(u) D u \cdot \gamma+2 C_{1}^{\prime}(u)\right] d x
\end{aligned}
$$




$$
=\int_{\Omega \times\{t\}}\left(-u_{t}^{2}+u_{t}\left[f \operatorname{div} \gamma+2 C_{1}^{\prime}(u)\right]\right) d x .
$$

Now we use the Sobolev-Orlicz imbedding theorem [2, Theorem 3.2(b)] and Cauchy's inequality to infer that $E^{\prime} \leq C$, and an approximation argument implies (2.8b) for the general case.

Note that the condition relating $f$ and $C_{1}$ is not used directly. For the purposes of Lemma 5.1, we could have assumed that $|f(z)| \leq C_{2}(|z|)$ for some increasing function $C_{2}$; however, the condition on $G \circ g^{-1}(|f(z)|)$ guarantees, via Young's inequality, that $E$ is bounded from below. This lower bound is crucial for our estimate of how quickly the function $U$ defined by

$$
U(t)=\int_{\Omega \times\{t\}}[S(D u)+G(|D u|)] d x
$$

can increase.

TheOrem 5.2. Let $u$ solve (5.1) and suppose conditions (0.8), (0.9), (2.2) and

$$
S(p) \geq 2 G(|p|)
$$

are satisfied. Suppose that there is an even $C^{1}$ function $C_{1}$, which is increasing on $(0, \infty)$, such that

$$
G \circ g^{-1}(|f(z)|)+G(z) \leq C_{1}(z),
$$

and let $k>0$. Then there are positive constants $C_{2}\left(k, C_{1}, \Omega\right)$ and $\eta\left(k, C_{1}, \Omega\right)$ such that (3.10) with $B>C_{2}$ implies (0.6).

Proof. By the Sobolev-Orlicz imbedding theorem, there is a constant $C_{0}(k, \Omega)$ such that $|u(x, \sigma)| \leq C_{0}$ for $x \in \Omega$. Hence

$$
\begin{aligned}
E(\sigma) & \leq \int_{\Omega \times\{t\}}\left[S(D u)+G(|D u|)+G \circ g^{-1}(|f(u)|)+2 C_{1}(u)\right] d x \\
& \leq 3 \int_{\Omega \times\{t\}}\left[S(D u)+C_{1}(u)\right] d x \leq 3 k+3 C_{1}\left(C_{0}\right)|\Omega|,
\end{aligned}
$$

while a similar argument gives $E(\tau) \geq \frac{B}{2}$. Choosing $C_{2}=8\left(k+C_{1}\left(C_{0}\right)|\Omega|\right)$ gives $E(\tau)-$ $E(\sigma) \geq C$ and then (0.6) follows from Lemma 5.1.

The slow growth case is more delicate. The correct ideas are (mostly) present in [9], but an error crept into the proof of [9, Lemma 4.2]: in the estimate of the boundary integral, all powers of $|u|$ should be increased by $p$. As we shall see in Theorem 5.4, [9, Lemma 4.3] (and hence Theorem 4.4 there) is correct. To give a correct statement and proof of the result in [9, Lemma 4.2], we begin with a technical lemma. As before, the results of this lemma are obvious when $g(\tau)=\tau^{m}$ for some $m \in(0, n-1)$.

Lemma 5.3. Let $g$ and $G$ satisfy conditions $(2.3 \mathrm{a}, \mathrm{b})$, and let $C_{1}$ and $\theta$ be positive constants with $\theta \leq \frac{1}{2}$. Then there is a positive $C_{2}$ determined only by $g, n, \theta$, and $C_{1}$ such that

$$
g \circ G^{-1}\left(\theta H(\theta z)+C_{1}\right) \leq \theta\left|H^{\prime}(\theta z)\right|+C_{2} \quad \text { for any real } z .
$$


Proof. We define $k=H^{-1}\left(\theta^{-n}+C_{1}(\theta) / \theta\right) / \theta$ and $C_{2}=g \circ G^{-1}\left(2 C_{1}+\theta^{1-n}\right)$ and note that $g \circ G^{-1}\left(\theta H(\theta z)+C_{1}\right) \leq C_{2}$ if $|z| \leq k$. Suppressing the argument $\theta z$ from $H$ and its derivatives, we have

$$
g \circ G^{-1}(2 \theta H) \leq g \circ G^{-1}(H)=\frac{H^{\prime}}{H^{1 / n}}
$$

if $z$ is positive. Because $H(\theta z) \geq \theta^{-n}$ if $z>k$, we have (5.9) in this case as well. Finally, (5.9) holds for $z<-k$ because $H$ is even.

For the slow growth case, our analysis is very close to that of Lemma 3.3.

LEMMA 5.4. Let $u$ be a solution of (5.1), suppose $E$ is given by (3.7), suppose (3.6) holds, and suppose there is a decreasing function $C_{1}$ such that

$$
G \circ g^{-1}(|f(z)|) \leq \theta H(\theta z)+C_{1}(\theta)
$$

for all real $z$ and all $\theta \in(0,1)$. If $B$ is a constant such that

$$
\|u(\cdot, t)\|_{H} \leq B
$$

for all $t \in\left[t_{0}, t_{1}\right]$, then there is a constant $\varepsilon_{0}=\varepsilon_{0}(\Omega, B)$ such that $\varepsilon \in\left(0, \varepsilon_{0}\right)$ implies that (2.8b) holds with $C$ determined only by $\varepsilon, B, C_{1}, \Omega, g_{1}$, and $g$.

Proof. By direct calculation, followed by use of the differential equation for $u$ and integration by parts, we have

$$
\begin{aligned}
E^{\prime}(t) & =\int_{\Omega \times\{t\}} H^{\prime} u_{t} d x=\int_{\Omega \times\{t\}} H^{\prime} \operatorname{div} A(D u) d x \\
& =-\varepsilon \int_{\Omega \times\{t\}} H^{\prime \prime} D u \cdot A(D u) d x-\varepsilon \int_{\partial \Omega \times\{t\}} H^{\prime} A(D u) \cdot \gamma d \sigma,
\end{aligned}
$$

where, as before, we suppress the argument of $H$ and its derivatives. Using the boundary condition and (5.10) yields

$$
-\int_{\partial \Omega \times\{t\}} H^{\prime} A(D u) \cdot \gamma d \sigma=\int_{\partial \Omega \times\{t\}} H^{\prime} f(u) d \sigma \leq \int_{\partial \Omega \times\{t\}}\left|H^{\prime}\right| g \circ G^{-1}\left(\varepsilon H+C_{1}\right) d \sigma .
$$

(The argument $\varepsilon$ is also suppressed from $C_{1}$.) Now we continue to estimate this integral. First, the divergence theorem gives

$$
\begin{aligned}
\int_{\partial \Omega \times\{t\}}\left|H^{\prime}\right| g \circ G^{-1}\left(\varepsilon H+C_{1}\right) d \sigma= & \int_{\Omega \times\{t\}}\left|H^{\prime}\right| g \circ G^{-1}\left(\varepsilon H+C_{1}\right) \operatorname{div} \gamma d x \\
& +\int_{\Omega \times\{t\}} \frac{d}{d u}\left(\left|H^{\prime}\right|\right) g \circ G^{-1}\left(\varepsilon H+C_{1}\right) D u \cdot \gamma d x \\
& +\int_{\Omega \times\{t\}}\left|H^{\prime}\right| \frac{d}{d u}\left(g \circ G^{-1}\left(\varepsilon H+C_{1}\right)\right) D u \cdot \gamma d x .
\end{aligned}
$$

To estimate these integrals, we first use (5.9) with $\theta=\varepsilon$ to see that

$$
g \circ G^{-1}\left(\varepsilon H+C_{1}\right) \leq \varepsilon\left|H^{\prime}\right|+C_{2} .
$$


Next, we have $\left|\frac{d}{d u}\right| H^{\prime}||=\varepsilon H^{\prime \prime}$, so

$$
\begin{aligned}
\left.\frac{d}{d u}\left(\left|H^{\prime}\right|\right) g \circ G^{-1}\left(\varepsilon H+C_{1}\right)\right) D u \cdot \gamma & \left.=\varepsilon H^{\prime \prime} g \circ G^{-1}\left(\varepsilon H+C_{1}\right)\right)|D u| \\
& \leq \varepsilon H^{\prime \prime} G(|D u|)+\varepsilon H^{\prime \prime}\left[\varepsilon H+C_{1}\right]
\end{aligned}
$$

by Young's inequality. Finally,

$$
\begin{aligned}
\frac{d}{d u}\left(g \circ G^{-1}\left(\varepsilon H+C_{1}\right)\right) & =\varepsilon^{2} g^{\prime}\left(G^{-1}\left(\varepsilon H+C_{1}\right)\right)\left(G^{-1}\right)^{\prime}\left(\varepsilon H+C_{1}\right) H^{\prime} \\
& =\frac{g^{\prime}\left(G^{-1}\left(\varepsilon H+C_{1}\right)\right)}{G^{\prime}\left(G^{-1}\left(\varepsilon H+C_{1}\right)\right)} \varepsilon^{2} H^{\prime} .
\end{aligned}
$$

Since $g$ and $G$ are increasing and $s g^{\prime}(s) \leq G^{\prime}(s)$, it follows that

$$
\frac{d}{d u}\left(g \circ G^{-1}\left(\varepsilon H+C_{1}\right)\right) \leq \frac{\varepsilon^{2}\left|H^{\prime}\right|}{G^{-1}\left(\varepsilon H^{\prime}+C_{1}\right)} .
$$

From this inequality and (3.2), we infer that

$$
\frac{d}{d u}\left(g \circ G^{-1}\left(\varepsilon H+C_{1}\right)\right)\left|H^{\prime}\right| D u \cdot \gamma \leq \frac{n \varepsilon^{2} H^{\prime \prime} H}{G^{-1}\left(\varepsilon H+C_{1}\right)}|D u| .
$$

Since $C_{1}$ is nonnegative, we infer from this inequality and Young's inequality that

$$
\frac{d}{d u}\left(g \circ G^{-1}\left(\varepsilon H+C_{1}\right)\right)\left|H^{\prime}\right| D u \cdot \gamma \leq n \varepsilon H^{\prime \prime} G(|D u|)+n \varepsilon H^{\prime \prime}\left[\varepsilon H+C_{1}\right] .
$$

Combining all these estimates and invoking (3.2) again yields

$$
E^{\prime}(t) \leq\left(C \varepsilon^{2}-\varepsilon\right) \int_{\Omega \times\{t\}} H^{\prime \prime} G(|D u|) d x+C \varepsilon \int_{\Omega \times\{t\}}\left(H^{\prime}\right)^{2} d x+C(\varepsilon) .
$$

Now an easy modification of Lemma 3.2 shows that

$$
\int_{\Omega \times\{t\}}\left(H^{\prime}\right)^{2} d x \leq C(\Omega) \varepsilon\left[\int_{\Omega \times\{t\}} H^{\prime \prime} G(|D u|) d x+\int_{\Omega \times\{t\}} H^{-1 / n}\left(H^{\prime}\right)^{2} d x\right]
$$

provided $\varepsilon \leq C\left(\Omega,\|u\|_{H}\right)$. Since $H^{-1 / n}\left(H^{\prime}\right)^{2}=C\left(g_{1}, n\right)|\varepsilon u|^{2-2 / n} \leq C$ for $|\varepsilon u| \leq 1$ and $H^{-1 / n}\left(H^{\prime}\right)^{2} \leq H(1)^{-1 / n}\left(H^{\prime}\right)^{2}$ otherwise, we infer (2.8b) for $\varepsilon$ sufficiently small.

Now the proof of Theorem 3.4 immediately gives the following growth rate estimate.

Theorem 5.5. Let $A$ and $g$ satisfy (2.3), (3.5) and (3.6), suppose that $f$ satisfies (5.10), suppose $u$ solves $(5.1)$, and set $U(t)=\|u(\cdot, t)\|_{H}$. If $B$ and $k$ are positive constants with $B>\max \{k, 8\}$, and if $\tau>\sigma$ are positive constants satisfying (3.10), then there is a positive constant $\delta$ determined only by $B, k, \Omega, C_{1}, g_{0}, g_{1}, n$, and $G$ such that (0.6) holds.

Note that when $A(p)=p$, we can use Theorem 5.5 in place of [9, Theorem 4.2] to prove [9, Lemma 4.3] and hence [9, Theorem 4.4].

For the borderline case, we note that (5.9) and Lemma 3.2, as well as the analog of this lemma which was used in Lemma 5.4, remain valid if

$$
g(z)=z^{n-1}, \quad H(z)=\exp \left(|z|^{n /(n-1)}\right) .
$$

Moreover, the case $n=2$ is included without any modifications. Hence the proof of Lemma 5.4 carries over to this case and we obtain the following results. 
Lemma 5.6. Suppose $E$ is given by (3.7) with $g$ and $H$ given by (5.12), and suppose there is a decreasing function $C_{1}$ such that (5.10) holds for all real $z$ and all $\theta \in(0,1)$. If $u$ is a solution of (5.1) and if $B$ is a constant such that (5.11) holds for all $t \in\left[t_{0}, t_{1}\right]$, then there is a constant $\varepsilon_{0}=\varepsilon_{0}(\Omega, B)$ such that $\varepsilon \in\left(0, \varepsilon_{0}\right)$ implies $(2.8 \mathrm{~b})$.

TheOREM 5.7. Let $A$ satisfy (3.5b) with $g$ and $H$ given by (5.12), suppose that $f$ satisfies (5.10), and set $U(t)=\|u(\cdot, t)\|_{H}$. If there are positive constants $B$ and $k$ with $B>\max \{k, 8\}$ and $\tau>\sigma$ satisfying (3.10), then there is a positive constant $\eta$ determined only by $B, k, \Omega, C_{1}, g_{0}, g_{1}, n$, and $G$ such that (0.6) holds.

Note that (5.10) with $g$ and $H$ given by (5.12) is equivalent to the condition

$$
|f(z)| \leq C_{3}(\theta) \exp \left(\theta|z|^{n /(n-1)}\right)
$$

for all real $z$, all $\theta \in(0,1)$, and a suitable function $C_{3}$.

6. Extensions of the conditions. There are several ways in which our results can be extended to cover more general situations by very minor modification of the arguments just presented. In this section, we discuss some of these extensions briefly.

First, many of our results are true if $A$ and $f$ are allowed to depend also on $x$ and $t$. For example, Theorem 2.1 holds with the additional hypothesis that $S_{t}(x, t, u, p) \leq$ $C_{3}[S(x, t, u, p)+1]$ for some constant $C_{3}$. Theorem 3.4 and 3.6 are true even without this additional assumption.

The most interesting extension is to the case that $A$ depends on $u$. In this case, our techniques seem to apply only when the equation can be written as

$$
u_{t}=\operatorname{div} A(D \Phi(u))+f(u, D u)
$$

with $A$ as before and $\Phi$ a strictly increasing $C^{1}(\mathbb{R} \backslash\{0\}) \cap C^{0}(\mathbb{R})$ function. Then Theorem 2.1 holds with

$$
\Phi^{\prime}(z) f(u, D u)^{2} \leq C_{0}(\Phi(z))+C_{1} S\left(\Phi^{\prime}(z) p\right)
$$

in place of (2.6) and $E$ defined by

$$
E(t)=\int_{\Omega \times\{t\}} S(D \Phi(u)) d x .
$$

This rapid growth case is virtually identical to the one in Section 2, but the slow growth case is more delicate. The results are easy to state; if there are positive constants $\theta_{1} \leq \theta_{2}$ such that

$$
H^{\prime}(s) \leq \theta_{1} s H^{\prime \prime}(s), \quad \theta_{1} \leq \frac{s \Phi^{\prime}(s)}{\Phi(s)} \leq \theta_{2}
$$

and if $f$ satisfies (3.9), then Theorems 3.4 and 3.6 are still true. To prove them in this case, we introduce a transformation $T$ defined by

$$
T[f](z)=\frac{1}{z} \int_{0}^{z} f(s) d s
$$

for $f \in L_{\text {loc }}^{1}(\mathbb{R})$ and set $\psi=T[T[\Phi]]$ and $h(s)=H(\varepsilon \psi(s))$ for $\varepsilon$ a positive constant at 
our disposal. A straightforward calculation, similar to one giving [10, (4.8)], shows that

$$
h^{\prime \prime} \geq \theta_{1}^{2} \varepsilon \max \left\{H^{\prime} \frac{\psi}{s^{2}}, \frac{\varepsilon}{\left(1+\theta_{1}\right)^{2}} H^{\prime \prime}\left(\frac{\psi}{s}\right)^{2}\right\} .
$$

Then the proof of Lemma 3.2 is easily modified to show that there are positive constants $K$ and $\varepsilon_{0}$ determined only by $\theta_{1}$ and $\theta_{2}$ such that

$$
\int_{\Omega}\left(h^{\prime}\right)^{2} d x \leq K \varepsilon \int_{\Omega} h^{\prime \prime} G(|D \Phi|) d x
$$

for $\varepsilon \leq \min \left\{1 /\|\Phi\|_{h}, \varepsilon_{0}\right\}$. From this inequality, the theorems follow just as before.

Finally, one can combine the techniques of Sections 2, 3, and 4 with those in Section 5 to consider problems of the form

$$
u_{t}=\operatorname{div} A(D u)+f(u, D u) \quad \text { in } \Omega \times(0, \infty), \quad A(D u) \cdot \gamma+F(u) \quad \text { on } \partial \Omega \times(0, \infty) .
$$

We refer the interested reader to [1] for details on this situation when $A(p)=p$ and $f$, which only depends on $u$, and $F$ are power functions.

\section{References}

[1] M. Chipot, M. Fila and P. Quittner, Stationary solutions, blow up and convergence to stationary solutions for semilinear parabolic equations with nonlinear boundary conditions, Acta Math. Univ. Comenian. 60 (1991), 35-103.

[2] T. K. Donaldson and N. S. Trudinger, Orlicz-Sobolev spaces and imbedding theorems, J. Funct. Anal. 8 (1971), 52-75.

[3] M. Fila, Boundedness of global solutions for the heat equation with nonlinear boundary conditions, Comm. Math. Univ. Carolin. 30 (1989), 479-484.

[4] —, Boundedness of global solutions of nonlinear diffusion equations, J. Differential Equations 98 (1992), 226-240.

[5] D. Gilbarg and N. S. Trudinger, Elliptic Partial Differential Equations of Second Order, 2nd ed., Springer, Berlin 1983.

[6] P. Grisvard, Elliptic Problems in Nonsmooth Domains, Pitman, London, 1985.

[7] M. A. Krasnosel'skii and Ya. B. Rutickii, Convex Functions and Orlicz Spaces, Noordhoff, Groningen, 1961.

[8] G. M. Lieberman, The natural generalization of the natural conditions of Ladyzhenskaya and Ural'tseva for elliptic equations, Comm. Partial Differential Equations 16 (1991), 311-361.

[9] - Study of global solutions of parabolic equations via a priori estimates I. Equations with principal elliptic part equal to the Laplacian, Math. Methods Appl. Sci. 16 (1993), 457-474.

[10] G. M. Lieberman, Study of global solutions of parabolic equations via a priori estimates II. Porous medium equations, Comm. Appl. Nonlinear Anal. 1 (1994), 93-115.

[11] - Maximum estimates for solutions of degenerate parabolic equations in divergence form, J. Differential Equations 113 (1994), 543-571.

[12] H. Matano, Asymptotic behavior of solutions of semilinear heat equations on $S^{1}$, in: Nonlinear Diffusion Equations and Their Equilibrium States, W.-M. Ni, L. A. Peletier and J. Serrin (eds.), Springer, 1988, 139-162. 
[13] L. M. Simon, Interior gradient bounds for non-uniformly elliptic equations, Indiana Univ. Math. J. 25 (1976), 821-855.

[14] N. S. Trudinger, On imbeddings into Orlicz spaces and some applications, J. Math. Mech. 17 (1967), 473-484.

[15] - An imbedding theorem for $H^{0}(G, \Omega)$ spaces, Studia Math. 50 (1974), 17-30.

[16] T. J. Zelenyak, Stabilization of solutions of boundary value problems for a second-order parabolic equation with one space variable, Differentsial'nye Uravneniya 4 (1968), 34-45; English transl.: Differential Equations 4 (1968), 17-22. 